\title{
Development of PCR Assays for Diagnosis and Detection of the Pathogens Phacidiopycnis washingtonensis and Sphaeropsis pyriputrescens in Apple Fruit
}

P. Sikdar, Department of Plant Pathology, Washington State University, Pullman 99164; P. Okubara, United States Department of Agriculture-Agricultural Research Service (USDA-ARS), Root Disease and Biological Control Research Unit, Pullman, WA 99164; M. Mazzola, USDA-ARS Tree Fruit Research Laboratory, Wenatchee, WA 98801; and C. L. Xiao, USDA-ARS San Joaquin Valley Agricultural Sciences Center, Parlier, CA 93648

\begin{abstract}
Speck rot caused by Phacidiopycnis washingtonensis and Sphaeropsis rot caused by Sphaeropsis pyriputrescens are two recently reported postharvest diseases of apple. Infection by these two pathogens occurs in the orchard but remains latent before harvest. Symptoms develop after harvest and are similar to those of gray mold caused by Botrytis cinerea. Accurate diagnosis of these diseases is important during the fruit inspection process, particularly in the instance of fruit destined for export. Early near-harvest detection of latent infections in apple fruit is an important step to implement relevant pre- and postharvest measures for disease control. The aim of this study was to develop polymerase chain reaction (PCR) assays for diagnosis and early detection of latent infections of apple fruit by $P$. washingtonensis and $S$. pyriputrescens. Species-specific primers based on the ribosomal DNA internal tran-
\end{abstract}

Abstract

Sikdar, P., Okubara, P., Mazzola, M., and Xiao, C. L. 2014. Development of PCR assays for diagnosis and detection of the pathogens Phacidiopycnis washingtonensis and Sphaeropsis pyriputrescens in apple fruit. Plant Dis. 98:241-246.

scribed spacer region were designed for use in PCR assays. Conventional and real-time PCR assays were developed and validated using fruit inoculated with $P$. washingtonensis, S. pyriputrescens, or B. cinerea and compared with identifications using traditional isolationbased assays. For wound-inoculated fruit, the PCR assays consistently provided the correct identification of the pathogen used as the inoculant in $6 \mathrm{~h}$ of processing time, compared with 5 to 6 days using culturebased methods. Real-time PCR assays effectively detected latent infections in symptomless stem and calyx tissues of fruit that were inoculated with the pathogens in the orchard during the growing season. The PCR assays provide a rapid, accurate method for diagnosis and early detection of these diseases.
Speck rot caused by Phacidiopycnis washingtonensis C.L. Xiao and J.D. Rogers (21) and Sphaeropsis rot caused by Sphaeropsis pyriputrescens C.L. Xiao and J.D. Rogers (20) are two recently described postharvest fruit rot diseases of apple $(6,8,14,17,21)$. Both diseases have the potential to cause significant economic losses of fruit during storage $(6,8)$. Initially, these two diseases were reported only in North America, and $P$. washingtonensis has since also been reported on apple in Germany and on persimmon in Italy (2,15). P. washingtonensis and S. pyriputrescens have been designated as quarantined pathogens by certain other countries.

Infections of apple fruit by $P$. washingtonensis and S. pyriputrescens occur in the orchard and remain latent at harvest, and symptoms develop during storage or in the market $(6,8)$. Stem-end rot and calyx-end rot are two major types of symptoms of speck rot and Sphaeropsis rot on apple fruit; the symptoms of these two diseases are very similar to those of gray mold caused by Botrytis cinerea, a major postharvest disease of apple; consequently, speck rot and Sphaeropsis rot are often misdiagnosed as gray mold (17).

Apple fruit are subjected to inspection for postharvest diseases prior to shipping to overseas markets. Similarity in symptoms among different diseases poses significant challenges to inspectors for accurate disease diagnosis. Traditional culture-based ap-

Corresponding author: C. L. Xiao, E-mail: Chang-Lin.Xiao@ars.usda.gov

Mention of trade names or commercial products in this article is solely for the purpose of providing specific information and does not imply recommendations or endorsement by the United States Department of Agriculture (USDA). USDA is an equal opportunity provider and employer.

Accepted for publication 3 September 2013.

http://dx.doi.org/10.1094/PDIS-05-13-0495-RE

This article is in the public domain and not copyrightable. It may be freely reprinted with customary crediting of the source. The American Phytopathological Society, 2014. proaches are reliable but labor intensive and time consuming, and require special skills in mycology. In contrast to conventional culture-based diagnosis methods, polymerase chain reaction (PCR) is a fast and less skill-intensive method for pathogen detection (11). PCR is now widely used in the identification of plant-pathogenic fungi primarily because detection and diagnosis are not dependent upon external symptom development. In addition, such a protocol can be utilized for early detection of latent pathogens (13), which would be of obvious benefit to growers and packers in determining the market destination of fruit, whether that be domestic or export.

The ribosomal DNA internal transcribed spacer region (ITS; 16) has been widely used as the target for development of speciesspecific PCR primers used in the detection of plant-pathogenic fungi (3). ITS regions are generally conserved within a particular species and variable among different fungal species and genera. Also, the ribosomal RNA genes are present in multiple copies, thus enhancing the likelihood of amplification relative to that of a single copy gene. Similar to traditional PCR, real-time PCR-based diagnosis is a fast and less skill-intensive tool for diagnosis of plant diseases. It also avoids the post-PCR steps and hazards relative to ethidium bromide staining in conventional PCR and allows accurate determination of pathogen DNA concentration in host tissues.

The objectives of this research were to develop PCR assays for (i) differential diagnosis of speck rot, Sphaeropsis rot, and gray mold on apple fruit and (ii) detection of latent infection in apple fruit caused by $P$. washingtonensis and S. pyriputrescens.

\section{Materials and Methods}

Fungal isolates and DNA isolation. Twelve isolates each of $B$. cinerea, S. pyriputrescens, and $P$. washingtonensis were included in this study (Table 1). Nontarget fungal pathogens known to cause postharvest diseases in apple and other nontarget fungi that are commonly associated with apple fruit were also included (Table 1). Fungal mycelia of these isolates previously stored in $15 \%$ glycerol at $-80^{\circ} \mathrm{C}$ were revived on potato dextrose agar (PDA; Difco Laboratory subsidiary of Becton, Dickinson and Company) and trans- 
ferred to potato dextrose broth (PDB; Difco Laboratory). The mycelium was grown on still culture plates of PDB at $20^{\circ} \mathrm{C}$ in the dark for 7 days. Mycelia from 7-day-old PDB cultures were used for DNA extraction using the FastDNA kit (MP Biomedicals) and the FastPrep-24 Instrument (MP Biomedicals).

Genomic DNA was extracted from diseased or symptomless apple fruit. Prior to extraction, apple fruit were surface sterilized with $70 \%$ ethanol and then allowed to air dry for 5 to $10 \mathrm{~min}$, and 100mg tissue samples were obtained from the stem and calyx end of the fruit. DNA was extracted using the same FastDNA kit (MP Biomedicals) with the modification of substituting CLS-TC (MP Biomedicals) and PPS (MP Biomedicals) as the lysis buffer in place of CLS-Y lysis buffer (MP Biomedicals). For hard stem tissues, two ceramic spheres were added to the lysis matrix (supplied in the kit) in the tube rather than one as outlined in the manufacturer's protocol. Tissues were incubated for $30 \mathrm{~min}$ before and after lysis in the provided tubes. DNA concentration was quantified using the Nanodrop 2000 spectrophotometer (Thermo Scientific). The concentration of DNA from mycelia was adjusted to10 $\mathrm{ng}^{-1}$ after measurement.

Table 1. List of target apple pathogens and nontarget fungi included in the polymerase chain reaction (PCR) and real-time PCR

\begin{tabular}{|c|c|}
\hline Fungal organism & Isolate number \\
\hline Botrytis cinerea & CLX 0193 \\
\hline B. cinerea & CLX 1760 \\
\hline B. cinerea & CLX 1924 \\
\hline B. cinerea & CLX 1881 \\
\hline B. cinerea & CLX 0288 \\
\hline B. cinerea & CLX 0859 \\
\hline B. cinerea & CLX 0823 \\
\hline B. cinerea & CLX 0377 \\
\hline B. cinerea & CLX 1756 \\
\hline B. cinerea & CLX 0458 \\
\hline B. cinerea & CLX 0473 \\
\hline B. cinerea & CLX 1940 \\
\hline Phacidiopycnis washingtonensis & CLX 2151 \\
\hline P. washingtonensis & CLX 2152 \\
\hline P. washingtonensis & CLX 2528 \\
\hline P. washingtonensis & CLX 6505 \\
\hline P. washingtonensis & CLX 2527 \\
\hline P. washingtonensis & CLX 4900 \\
\hline P. washingtonensis & CLX 2520 \\
\hline P. washingtonensis & CLX 2521 \\
\hline P. washingtonensis & CLX 4928 \\
\hline P. washingtonensis & CLX 7683 \\
\hline P. washingtonensis & CLX 5166 \\
\hline P. washingtonensis & CLX 6495 \\
\hline Sphaeropsis pyriputrescens & CLX 2380 \\
\hline S. pyriputrescens & CLX 3026 \\
\hline S. pyriputrescens & CLX2044 \\
\hline S. pyriputrescens & CLX 3002 \\
\hline S. pyriputrescens & CLX 4941 \\
\hline S. pyriputrescens & CLX 2533 \\
\hline S. pyriputrescens & CLX 4496 \\
\hline S. pyriputrescens & CLX 3006 \\
\hline S. pyriputrescens & CLX 6453 \\
\hline S. pyriputrescens & CLX 1022 \\
\hline S. pyriputrescens & CLX 3001 \\
\hline S. pyriputrescens & CLX 4495 \\
\hline Mucor piriformis & CLX 4950 \\
\hline M. piriformis & CLX 3820 \\
\hline Alternaria alternata & CLX 8238 \\
\hline A. alternata & CLX 8249 \\
\hline Penicillium expansum & CLX 3472 \\
\hline P. expansum & CLX 3292 \\
\hline Phacidiopycnis piri & CLX 206 \\
\hline P. piri & CLX 210 \\
\hline Cladosporium herbarum & CLX 8236 \\
\hline C. herbarum & CLX 5162 \\
\hline Neofabraea alba & CLX 4130 \\
\hline N. perennans & CLX 7054 \\
\hline Aureobasidium pullulans & CLX 8240 \\
\hline Cytospora sp. & CLX 3269 \\
\hline
\end{tabular}

Primer design and annealing temperature determination. ITS sequences of the three target pathogens and eight nontarget fungi were obtained from GenBank (Table 2). Multiple alignments of all sequences were performed using CLUSTALW online (European Molecular Biology Laboratory-European Bioinformatics Institute [EMBL-EBI]; 4,9). Specific primer sets for $P$. washingtonensis, $S$. pyriputrescens, and $B$. cinerea were designed using web-based primer design software at the Saccharomyces Genome Database (http://www.yeastgenome.org/cgi-bin/web-primer). One pair of species-specific primers was designed for each target pathogen (Table 3). To determine the annealing temperature of each primer pair, temperature gradient PCR was carried out using an annealing temperature of 50 to $60^{\circ} \mathrm{C}$. The isolates $B$. cinerea CLX0377, P. washingtonensis CLX2152, and S. pyriputrescens CLX2380 were used for this initial PCR. For each primer pair, PCR was carried out in a 25 - $\mu$ l reaction volume containing $2 \mathrm{ng}$ of fungal genomic DNA, $0.4 \mu \mathrm{M}$ each primer, $0.2 \mathrm{mM}$ dNTP (Promega Corporation), $1 \times$ Go Taq Buffer (Promega Corporation), $2.0 \mathrm{mM} \mathrm{MgCl}{ }_{2}$ (Promega Corporation), and $1.25 \mathrm{U}$ of Go Taq DNA Polymerase (Promega Corporation). PCR was conducted using an iCycler thermocycler (Bio-Rad) with the following parameters: an initial heating step at $95^{\circ} \mathrm{C}$ for $3 \mathrm{~min}$; followed by 35 cycles of denaturation at $94^{\circ} \mathrm{C}$ for $30 \mathrm{~s}$, annealing at temperature gradient of 50 to $60^{\circ} \mathrm{C}$ for $30 \mathrm{~s}$, and extension at $72^{\circ} \mathrm{C}$ for $30 \mathrm{~s}$; and final extension at $72^{\circ} \mathrm{C}$ for $10 \mathrm{~min}$. The PCR products were separated on $1 \%$ agarose gels in Tris-acetate-EDTA buffer, stained using ethidium bromide, and photographed using a UVP GelDoc-It Imaging System (Ultra Violet Products). Optimal annealing temperatures are given in Table 3 .

Conventional PCR for the diagnosis of three pathogens. Conventional PCR was conducted to test the specificity of primers using DNA from 12 isolates of each of the target pathogens ( $B$. cinerea, $P$. washingtonensis, and $S$. pyriputrescens) and 1 to 2 isolates of each nontarget fungi (Table 1). The PCR reaction was carried out using the same reagent concentrations as described above. Other than the use of the optimized annealing temperature (Table 3 ) for the respective primer pairs, the amplification conditions were the same as noted previously. Following PCR, amplicons were separated via gel electrophoresis and visualized as described above.

Sensitivity, specificity, and efficiency of real-time PCR assays. Standard curves plotting cycle threshold $(\mathrm{Ct})$ value (y-axis) versus the log of the DNA concentration ( $\mathrm{x}$-axis) were generated to estimate the sensitivity of the primer pairs designed for $B$. cinerea, $P$. washingtonensis, and $S$. pyriputrescens. Each standard curve was constructed from seven 10-fold dilutions of DNA extracted from pure fungal cultures, with DNA concentrations of $5 \mathrm{ng} \mathrm{\mu l}^{-1}$ to $5 \mathrm{fg} \mathrm{l}^{-1}$.

Studies were conducted to determine whether plant extracts could potentially inhibit the PCR when species-specific primers were utilized. DNA was extracted from healthy organic Fuji apple tissue using the FastDNA kit (MP Biomedicals) as described in the

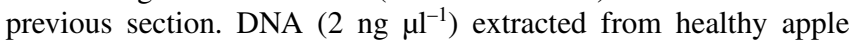
fruit tissues was spiked with DNA from fungal pure cultures $(5 \mathrm{ng}$

Table 2. Fungi included in the multiple sequence alignment study and their corresponding GenBank number

\begin{tabular}{lc}
\hline Pathogen & GenBank accession number \\
\hline Alternaria alternata & GU062279 \\
Aureobasidium pullulans & JN585936 \\
Botrytis cinerea & HM235973 \\
Cladosporium herbarum & AY354234 \\
Cytospora sp. & FJ903365 \\
Mucor pyriformis & EU484276 \\
Neofabraea alba & FJ228196 \\
Neofabraea perennans & AF281396 \\
Penicillium expansum & FJ491786 \\
Phacidiopycnis piri & AY608638 \\
P. washingtonensis & AY608644 \\
Sphaeropsis pyriputrescens & GQ374241 \\
\hline
\end{tabular}


$\mu \mathrm{l}^{-1}$ to $5 \mathrm{fg} \mu \mathrm{l}^{-1}$ ) of each pathogen, to generate apple extract standard curves. In addition, real-time PCR standard curves were generated using primers designed for the three pathogens and healthy apple genomic DNA as template with concentrations from $5 \mathrm{ng} \mathrm{\mu l}^{-1}$ to $5 \mathrm{fg} \mathrm{l}^{-1}$. The efficiency $(E)$ of target amplification in the presence of the host extract was calculated using the equation $E=10^{1 /-m}-1 \times$ $100(\mathrm{~m}=$ slope of standard curve) $(5)$, and compared with that for standard curves generated with DNA diluted in water.

Similar to conventional PCR, primer specificity was also tested in real-time PCR using target pathogen and nontarget fungal DNA (Table 1). PCR was carried out in 20- $\mu$ l reaction volumes containing $1 \times$ iQ SYBR Green Supermix (Bio-Rad), $150 \mathrm{nM}$ forward primer, $150 \mathrm{nM}$ reverse primer, $0.5 \mathrm{ng}$ of DNA, and sterile deionized water $\left(\mathrm{dH}_{2} \mathrm{O}\right)$. PCR was carried out in an iQ5 iCycler Thermocycler (Bio-Rad) with the following parameters: an initial heating at $95^{\circ} \mathrm{C}$ for $3 \mathrm{~min}$ and 40 cycles of denaturation at $95^{\circ} \mathrm{C}$ for $30 \mathrm{~s}$, annealing at respective annealing temperature of each primer set (Table 3 ) for $30 \mathrm{~s}$, and extension at $72^{\circ} \mathrm{C}$ for $30 \mathrm{~s}$. To determine the melting temperature $(\mathrm{Tm})$ of the amplicon, fluorescence data were collected after the annealing step in each cycle. Melting curves were obtained for the amplicons that were generated by raising the reaction temperature from 50 to $90^{\circ} \mathrm{C}$ in $0.1^{\circ} \mathrm{C}$ increments, with fluorescence acquired every $0.5 \mathrm{~s}$. All fluorescence data $(\mathrm{Ct}$ values and amplicon $\mathrm{Tm})$ were analyzed using iQ Software (Bio-Rad).

Validation of real-time PCR assays using laboratory inoculated apple fruit. Ninety organic 'Fuji' apple fruit were selected and then divided into three sets of 30 apple fruit in each set. Each set of 30 fruit was individually inoculated with one of the test fungal pathogens: $P$. washingtonensis CLX 2152, B. cinerea CLX0377, or S. pyriputrescens CLX2380. For inoculum preparation, the pathogen isolates were revived on PDA from glycerol stock stored at $-80^{\circ} \mathrm{C}$. After 5 to 7 days of incubation at $20^{\circ} \mathrm{C}$ in the dark, resulting spores were streaked onto oatmeal agar medium (made by adding $20 \mathrm{~g}$ of Gerber Oatmeal Cereal and $5 \mathrm{~g}$ of agar [Difco Laboratory] in 1 liter of sterile $\mathrm{dH}_{2} \mathrm{O}$ [autoclaved twice for $45 \mathrm{~min}]$ ) and the cultures were incubated at $20^{\circ} \mathrm{C}$ with 12 -h alternating light and dark cycles for 5 to 6 days. Conidia from these plates were used to prepare inocula in sterile $\mathrm{dH}_{2} \mathrm{O}$. The final spore concentration was adjusted to $5 \times 10^{5}$ conidia $\mathrm{ml}^{-1}(7,10)$. The fruit were surface disinfected in a $0.5 \% \mathrm{NaOCl}$ solution for $5 \mathrm{~min}$ and then rinsed three times with sterile water and allowed to dry for 1 h. An artificial wound was made on each apple fruit with a sterile 2-mm-diameter finish nail head to a depth of $2 \mathrm{~mm}$. Each wound was inoculated with $20 \mu \mathrm{l}$ of the conidial suspension $\left(5 \times 10^{5}\right.$ conidia $\mathrm{ml}^{-1}$ ). The fruit were then transferred to crispers, and humidity in each crisper was maintained with moistened paper towels. Crispers were incubated at room temperature in the dark for 7 to 10 days until rot developed. The reason that the fruit were stored at room temperature rather than at cold $\left(0\right.$ to $\left.4^{\circ} \mathrm{C}\right)$ temperatures commonly used commercially for storage of apple fruit was to facilitate the development of decay symptoms on inoculated apple fruit so that the decayed tissues could be used for PCR assays, because it takes longer for the inoculated fruit to develop decay symptoms at cold temperatures. The specific goal of this experiment was to validate the PCR assays using decayed apple tissues resulting from inoculation, not to compare how storage temperatures affect the progress of symptom development on apple fruit. DNA was extracted from the decayed tissue and real-time PCR was conducted as described above. To isolate causal agents, a piece of symptomatic tissue was excised from each fruit using a sterile scalpel and plated on PDA, and plates were incubated in the dark at $20^{\circ} \mathrm{C}$ for 7 days. Fungi were identified based upon cultural characteristics and compared with the real-time PCR data. Results obtained via real-time PCR assays and apple extract standard curves were compared with those acquired using conventional isolationbased protocols.

In a second experiment, 90 organic 'Red Delicious' apple fruit were assigned a number and then 30 fruit each were randomly infected with $P$. washingtonensis, B. cinerea, or $S$. pyriputrescens. After inoculation, the apple fruit were shuffled randomly and subsequently divided into three groups with their assigned numbers removed. The fruit were inoculated and incubated in a similar manner as described above. After 15 days of incubation, DNA was extracted from the infected tissue, real-time PCR was conducted, and results were compared with diagnoses obtained using conventional isolation methods.

Detection of $P$. washingtonensis and $S$. pyriputrescens latent infection. This experiment was conducted in a Red Delicious block located at the Washington State University Sunrise research orchard near Palisades, Douglas County (latitude $47.3^{\circ} \mathrm{N}$ and longitude $120.1^{\circ} \mathrm{W}$ ). Fruit inoculation in situ was conducted twice during the 2011 growing season. The initial inoculation was conducted in the last week of July and the second inoculation took place during the first week of September. Inocula were prepared as described above. Fruit were inoculated by spraying until runoff with spore suspensions of $5 \times 10^{5}$ conidia $\mathrm{ml}^{-1}$ of either $P$. washingtonensis CLX2152 or S. pyriputrescens CLX2380 (10). In total, 60 fruit (three replicates with 20 fruit per replicate) were inoculated with each isolate. All inoculations were conducted near sunset, and fruit were covered and incubated in moist plastic bags for $16 \mathrm{~h}$. To avoid direct sunlight, white paper was attached around the bags. The fruit were harvested 2 weeks prior to commercial harvest date in September 2011 and assayed for the respective pathogens to determine sensitivity of the real-time PCR assay for detection of latent infections. All fruit were surface sterilized with $70 \%$ ethanol at the stem-end and calyx-end of the fruit and then air dried for 5 to 10 min before extraction of DNA or isolation from asymptomatic tissue on PDA. DNA extraction from symptomless stem and calyx tissue immediately after harvest and real-time PCR analysis of the resulting DNA extracts were conducted as described above. The sensitivity of the PCR assay was compared with that of traditional culture-based methods by conducting isolations for the respective pathogens from inoculated tissues on PDA.

Data analysis. For obtaining the slope of real-time PCR standard curves, DNA concentration was log transformed and then plotted against the $\mathrm{Ct}$ value on the $\mathrm{y}$-axis. Efficiency of the assay was calculated by using the formula $E=10^{(1 / \text {-slope })}-1 \times 100$, based on Ginzinger (5). All calculations were conducted using Microsoft Excel from Microsoft Office (2010 edition). To assess whether the two diagnostic methods differed significantly in efficiency of pathogen diagnosis, data for latent and symptomatic infections as determined by culture-based and PCR-based methods were analyzed using Student's $t$ test performed with SAS PROC TTEST (version

Table 3. Primers, optimized annealing temperature, size of product, and melting curve peak

\begin{tabular}{llccc}
\hline Primer name & \multicolumn{1}{c}{ Primer sequence } & Annealing temperature $\left({ }^{\circ} \mathbf{C}\right)$ & Size of product $(\mathbf{b p})$ & Product melt curve peak $\left({ }^{\circ} \mathbf{C}\right)$ \\
\hline P.W FP2 & CTCCAAAACTCATATTGTCAT & 54 & $\ldots$ & $\ldots$ \\
P.W RP1 & CTGAAAAAATATGGGGGTTT & 54 & 351 & 88 \\
B.C FP1 ${ }^{y}$ & TGTATGCTCGCCAGAGAAAA & 58 & $\ldots$ & $\ldots$ \\
B.C RP1 & AGCACACCGAGAACCTGTAA & 58 & 344 & 86 \\
S.P FP1 $1^{z}$ & AGGACTCCAAAACCCATATC & 58.6 & $\ldots$ & $\ldots$ \\
S.P RP1 & TATTACTACGCTTGAAGCCAG & 58.6 & 301 & 87 \\
\hline
\end{tabular}

${ }^{x}$ P.W FP2 and P.W RP1 are species-specific primers for Phacidiopycnis washingtonensis.

${ }^{y}$ B.C FP1 and B.C RP1 are species-specific primers for Botrytis cinerea.

${ }^{z}$ S.P FP1 and S.P RP1 are species-specific primers for Sphaeropsis pyriputrescens. 
9.3.3; SAS Institute). Percentage data were square root transformed prior to $t$ test analysis.

\section{Results}

General PCR. The PCR primer pairs designed to specifically target each of the three fungal pathogens examined in this study, their corresponding amplicon size, and Tm and annealing temperatures are listed in Table 3. Each primer pair exclusively amplified the ITS region of the 12 isolates of the specific target pathogen (data not shown). Using these primer pairs, no cross amplification was observed with the other two target pathogens and no amplification product was obtained when DNA from nontarget fungi was included in the amplification reaction (data not shown).

Sensitivity of primer pairs and efficiency of real-time PCR assays. Using the respective species-specific primers, the detection limit for P. washingtonensis, S. pyriputrescens, and B. cinerea in
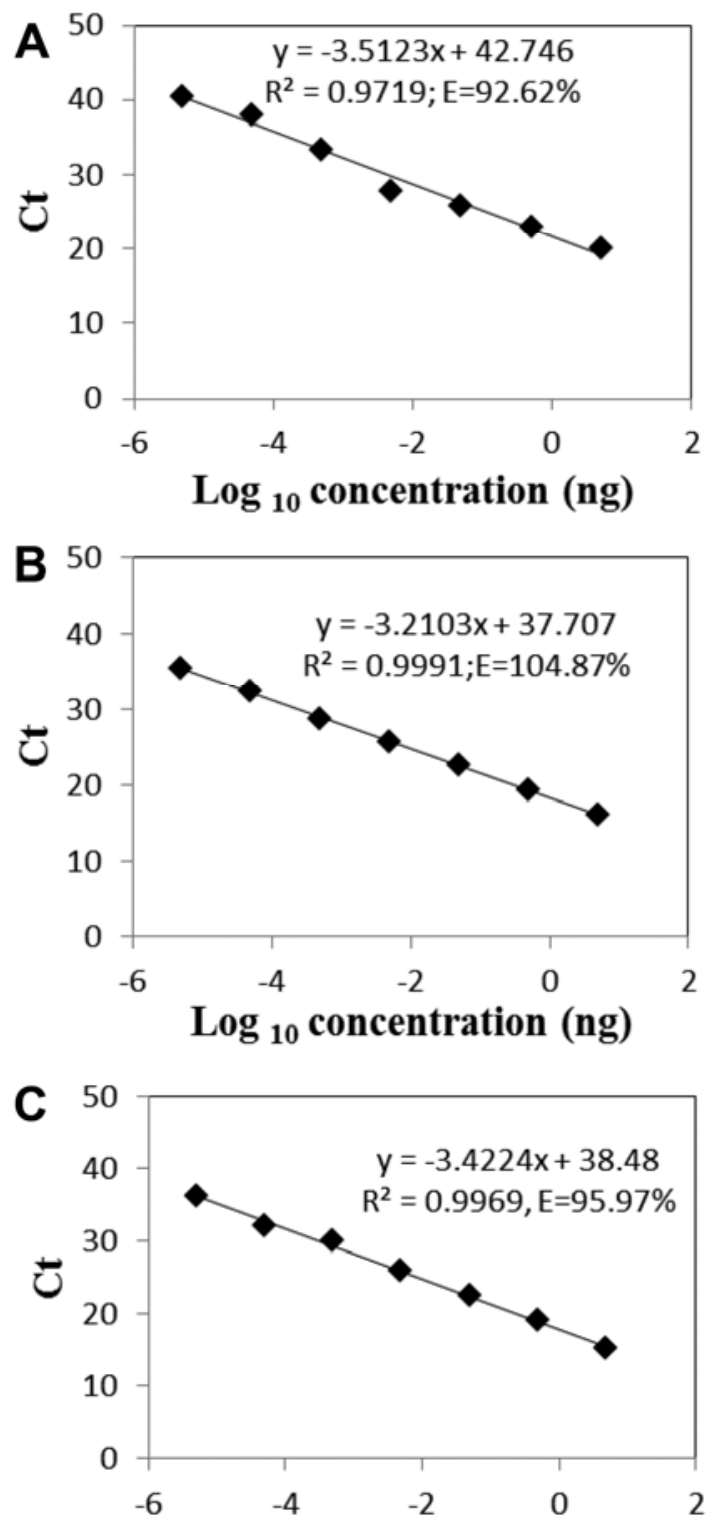

$\log _{10}$ concentration (ng)

Fig. 1. Standard curves generated using genomic DNA from cultured fungi and real-time polymerase chain reaction (PCR) for each target postharvest pathogen species-specific primer set developed for identification and quantification. A range of DNA concentrations from $5 \mathrm{ng} \mathrm{\mu l}^{-1}$ to $5 \mathrm{fg}^{-1}{ }^{-1}$ was used in generating the above graphs. Efficiency $(E)$ of the real-time PCR reaction $=10^{1 /-m}-1 \times 100(\mathrm{~m}=$ slope of standard curve); $\mathrm{Ct}=$ cycle threshold. $\mathbf{A}$, Phacidiopycnis washingtonensis primer set P.W FP2/ P.W RP1; B, Botrytis cinerea primer set B.C FP1/B.C RP1; and C, Sphaeropsis pyriputrescens primer set S.P FP1/ S.P RP1. the real-time PCR assays was $5 \mathrm{fg}$, with the lowest $\mathrm{Ct}$ value of 19 (Fig. 1). The limit of detection for all three test fungi using DNA extracted from apple tissues was $50 \mathrm{fg}$, compared with $5 \mathrm{fg}$ for DNA from pure cultures. The cut-off $\mathrm{Ct}$ value for all assays was 35. There was no amplification using any of the three primers designed for target pathogens (i.e., P. washingtonensis, S. pyriputrescens, and B. cinerea) for real-time PCR conducted only with genomic DNA extracted from healthy apple tissue (data not shown). The efficiencies of real-time PCR assays calculated using purified fungal DNA in water or the same DNA extract spiked with apple tissue extract were similar for all three fungal pathogens (data not shown). For a particular primer set, there was no cross reaction noted for either of the other two target pathogens and no signals were obtained in reactions using DNA extracted from any of the nontarget fungi or apple tissue.

Validation of real-time PCR assay with laboratory-inoculated apple fruit. Fuji apple fruit from the first experiment developed spongy to firm rot at the point of inoculation within 10 days when incubated at room temperature. Using DNA extracted from the diseased fruit flesh, real-time PCR successfully identified the causal pathogens. There was a $100 \%$ correlation between identifications obtained using the real-time PCR assays and those obtained using culture-based methods (Table 4). During the postinoculation period, some of the fruit became contaminated with Penicillium spp., which was apparent under visual inspection. Among these fruit, one, two, and five in the group preinoculated with $P$. washingtonensis, $S$. pyriputrescens, or $B$. cinerea, respectively, became contaminated with Penicillium spp. All fruit that exhibited decay by Penicillium spp. did not yield specific PCR products in real-time PCR.

Because the symptoms of the three postharvest diseases are similar, the second experiment was designed to simulate the presence of all three diseases occurring simultaneously within a fruit lot in a packing house, as a further test for the specificity of the assays. For each replicated set of fruit, the real-time PCR assays provided the same pathogen identifications as those determined by isolations on PDA (Table 5). However, the PCR assays were superior in being able to provide an accurate identification in only $6 \mathrm{~h}$, whereas the same identification required 5 to 6 days using the culture-based method.

Detection of latent $P$. washingtonensis and $S$. pyriputrescens infections at harvest. The real-time PCR assay detected $P$. washingtonensis in symptomless tissue for 85 and $90 \%$ of the fruit inoculated in July and September, respectively. In contrast, the pathogen was identified from only $79.2 \%$ (July) and $84.2 \%$ (September) of the same tissue using conventional isolation methods on PDA. For S. pyriputrescens, 95 and $100 \%$ of latent infections were detected by real-time PCR from the fruit inoculated in July and September, respectively, while $90 \%$ of latent infections were detected using the culture-based method. When all (July and September) data were considered, real-time PCR detected P. washingtonensis from $87.5 \%$ of total symptomless inoculated fruit, which was significantly higher $(P=0.011)$ than that determined using conventional isolation methods (81.6\%; Table 6). However, when a $t$ test was carried out individually for symptomless stem tissues and calyx-end tissues, real-time PCR detected a significantly $(P=0.015)$ higher percentage of stem latent infections than did the culture-based assay but detection rates from calyx tissues were not significantly different $(P=0.14)$ between the two methods (Table 6). Similarly, the real-time PCR method detected $S$. pyriputrescens latent infections at a significantly $(P=0.001)$ higher rate than the culture-based method and, though stem latent infections were detected at a significantly $(P=0.001)$ higher rate by the real-time PCR method, there was no significant $(P=0.16)$ difference between the two methods in detecting calyx-end infections (Table 6).

\section{Discussion}

Conventional and real-time PCR assays were developed for the efficient diagnosis of speck rot, Sphaeropsis rot, and gray mold, 
and at-harvest early detection of latent infections in apple fruit by $P$. washingtonensis and S. pyriputrescens. This is the first report of PCR assays that distinguish the pathogens inciting gray mold, Sphaeropsis rot, and speck rot in apple fruit. In our study, the species-specific primers were developed based on the ITS region. In general, multiple genes may need to be sequenced for speciesspecific primer development. Initially, we sequenced the intergenic spacer (IGS) region of the three pathogens because a real-time PCR assay has been developed for the detection of $B$. cinerea in onion using $B$. cinerea-specific primers based on the IGS region (1). However, we were unsuccessful in our attempt to utilize the same region to develop primers because there is insufficient polymorphism in the IGS region to differentiate among the three pathogens. Thus, we attempted to use the ITS region and, with the polymorphisms present, we were able to design primers based on the ITS regions of the pathogens that were robust to differentiate the three diseases and also detect latent infections in symptomless tissues. In conventional PCR assays, each primer pair efficiently amplified the ITS region for the specified fungal pathogen but did not amplify DNA from the other target pathogens. This is of significance because the ITS region of the three fungi possessed very little polymorphism. In an earlier publication, it was reported that the ITS region of $P$. washingtonensis differed from Potebniamyces pyri (anamorph Phacidiopycnis piri) by only 4 nucleotides (21). The PCR assays developed here possessed enough stringency to only amplify $P$. washingtonensis and not $P$. piri. The ITS-based primers met our needs; thus, we did not attempt to clone other genomic regions.

The conventional PCR assay had a detection limit of $5 \mathrm{ng}$ of target DNA for each of the respective target fungal pathogens. This was sufficient for diagnosis of the pathogen in fruit expressing visible symptoms of infection. However, latent fruit infections will possess a substantially lower density of the fungal pathogen and conventional PCR is unlikely to consistently and accurately detect the presence of the pathogen in symptomless apple fruit at harvest. The real-time PCR assays developed in this study detected the target pathogens in amounts as low as $5 \mathrm{fg}$ from pure cultures and $50 \mathrm{fg}$ from extracts of diseased tissue. The difference in detection has been observed in other types of plant samples (12), and might be due to the complexity of DNAs found in natural samples. Also, inhibitors naturally present in the harder plant tissues, such as in pedicels, can affect amplification efficiency. However, the detection limits of our assays were low enough to reliably detect the causal organism in infested tissues not yet expressing symptoms.

In the first experiment, a few fruit became inadvertently contaminated by Penicillium spp. and, consequently, typical blue mold symptoms developed on the fruit. None of the target pathogens $(S$. pyriputrescens, $P$. washingtonensis, and $B$. cinerea) used for inoculation was recovered from decayed tissues in culture-based assays. The real-time PCR assay with the decayed tissues from these blue mold-decayed fruit did not generate PCR products specific for $P$. washingtonensis, $S$. pyriputrescens, or $B$. cinerea, suggesting that false positives were avoided in these situations where the decay was caused by a nontarget pathogen.

Pathogens such as S. pyriputrescens and P. washingtonensis, which possess the capacity to incite latent fruit infections, have the potential to pose significant threats as high-risk postharvest pathogens if not controlled in time. In a survey conducted between 2003 and 2005, S. pyriputrescens was shown to be one of the most significant postharvest pathogens in Washington State (8). S. pyriputrescens was detected in all regions and $78 \%$ of the grower lots sampled, accounting for an average of $17 \%$ of decayed fruit (8). This figure places $S$. pyriputrescens among the top three postharvest pathogens of stored apple fruit in Washington along with $B$. cinerea and Penicillium expansum, which are responsible for 28.1 and $31.7 \%$ of decay, respectively. Speck rot caused by Phacidiopycnis washingtonensis occurred in $26 \%$ of the grower lots surveyed from 2003 to 2005 and accounted for $4 \%$ of total decay. However, in one particular grower lot sampled during 2004, this fungus incited decay in $24 \%$ of the stored fruit (6). Thus, $P$. wash-

Table 6. Percentage of Phacidiopycnis washingtonensis and Sphaeropsis pyriputrescens latent infections detected by real-time polymerase chain reaction (PCR) and culture-based isolation methods ${ }^{\mathrm{z}}$

\begin{tabular}{lccc}
\hline Pathogen, method & $\begin{array}{c}\text { Mean (all } \\
\text { sample tissues) }\end{array}$ & $\begin{array}{c}\text { Mean } \\
\text { (stem) }\end{array}$ & $\begin{array}{c}\text { Mean } \\
\text { (calyx) }\end{array}$ \\
\hline $\begin{array}{l}\text { P. washingtonensis } \\
\text { Real-time PCR }\end{array}$ & $87.5 \mathrm{a}$ & $88.3 \mathrm{a}$ & $86.6 \mathrm{a}$ \\
$\begin{array}{l}\text { Culture-based } \\
\text { S. pyriputrescens }\end{array}$ & $81.6 \mathrm{~b}$ & $82.5 \mathrm{~b}$ & $80 \mathrm{a}$ \\
$\begin{array}{l}\text { Real-time PCR } \\
\text { Culture-based }\end{array}$ & $97.7 \mathrm{a}$ & $98.3 \mathrm{a}$ & $93.3 \mathrm{a}$ \\
\hline
\end{tabular}

${ }^{\mathrm{z}}$ Means in a column followed by the same letter are not significantly different based upon the Student's $t$ test.

Table 4. Comparison of real-time polymerase chain reaction (PCR) and conventional isolation-based detection of the target pathogens using inoculated apple fruit ${ }^{\mathrm{y}}$

\begin{tabular}{lccc}
\hline Pathogen & $\begin{array}{c}\text { Number of } \\
\text { samples }\end{array}$ & $\begin{array}{c}\text { Number of diseased fruit diagnosed } \\
\text { by conventional method }\end{array}$ & $\begin{array}{c}\text { Number of diseased fruit diagnosed } \\
\text { by real-time PCR }(\text { Ct range })^{\mathbf{z}}\end{array}$ \\
\hline Phacidiopycnis washingtonensis & 30 & 29 & $29(17.5-21)$ \\
Botrytis cinerea & 30 & 25 & $25(15-20.5)$ \\
Sphaeropsis pyriputrescens & 30 & 28 & $28(14.6-22)$ \\
\hline
\end{tabular}

${ }^{y}$ Number of fruit exhibiting the symptoms of diseases incited by the respective pathogens used for inoculation; a few fruit were also contaminated by Penicillium spp.

${ }^{\mathrm{z}} \mathrm{Ct}=$ cycle threshold.

Table 5. Comparison of real-time polymerase chain reaction (PCR) and conventional isolation-based diagnosis for detection of the target fungi from apple fruit randomly inoculated in the laboratory ${ }^{z}$

\begin{tabular}{lclcc}
\hline Sample & Number of fruit & \multicolumn{1}{c}{ Causal agent } & Pathogen isolated on PDA & Real-time PCR $($ Ct range) \\
\hline Set 1 & \multirow{2}{*}{30} & Phacidiopycnis washingtonensis & 8 & $8(19.36-30.46)$ \\
& & Botrytis cinerea & 8 & $8(21.94-27.36)$ \\
Set 2 & \multirow{3}{*}{30} & Sphaeropsis pyriputrescens & 14 & $14(19.65-29.64)$ \\
& & P. washingtonensis & 11 & $11(21.245-28.99)$ \\
Set 3 & \multirow{2}{*}{30} & B. cinerea & 10 & $10(22.69-27.78)$ \\
& & S. pyriputrescens & 9 & $9(17.685-21.73)$ \\
& & P. washingtonensis & 11 & $11(20.68-28.10)$ \\
& & S. cinerea & 12 & $12(16.59-29.78)$ \\
\end{tabular}

${ }^{\mathrm{z}} \mathrm{PDA}=$ potato dextrose agar and $\mathrm{Ct}=$ cycle threshold 
ingtonensis has demonstrated the potential to become an important postharvest pathogen in Washington State. Because P. washingtonensis and $S$. pyriputrescens have been designated as quarantine pathogens in certain countries, stringent inspection for these two diseases is being implemented by inspectors for exports in order to reduce losses resulting from rejections of shipments with these diseases. The accurate diagnosis of the causal pathogens among a group of pathogens causing similar symptoms that is enabled by the PCR assays developed in this study will facilitate inspection of apple fruit for exports to overseas where these pathogens are quarantined.

The key step for implementation of effective measures to control postharvest diseases resulting from latent infections is accurate early detection of infections. Infection of apple fruit by $P$. washingtonensis and $S$. pyriputrescens occurs during the fruit growing season in the field, rather than during storage, with the stem and floral parts of the fruit serving as the primary infection courts $(6,7)$. In our study, fruit inoculations carried out in July and September with harvest 2 weeks before the commercial harvest demonstrated that the target pathogens could be successfully detected in fruit prior to harvest using the real-time PCR assays developed in the present study. This assay detected both $P$. washingtonensis and $S$. pyriputrescens from totally symptomless preinoculated fruit samples at a significantly higher percentage than the diagnosis performed using the conventional isolation-based methods. Percent detection of latent pathogens from the symptomless stem tissues was higher when real-time PCR assays were used for diagnosis. However, there was no statistically significant difference when an assay was done using the calyx tissues compared with the conventional method. Nonetheless, the real-time PCR assays were more sensitive than the conventional assays for the early detection of latent infections of apple fruit by $P$. washingtonensis and S. pyriputrescens. We also need to point out that the specific purpose of this experiment was to test whether the PCR assays, compared with the traditional isolation assay, are able to detect latent infections in fruit caused by the two pathogens. Because our results from the two separate experiments yielded consistent results in detecting latent infections in symptomless tissues of apple fruit, we did not repeat this experiment for another year, though we agree that, in general, more data points would be better for validation of the assays.

Chemical application is still the primary method utilized for the control of postharvest diseases of pome fruit in Washington State. Fungicides may be applied in the orchard before harvest or as a postharvest treatment at the storage and packing facility (18). Most pome fruit postharvest pathogens, including $S$. pyriputrescens and $P$. washingtonensis, can be controlled by application of fungicides as a postharvest drench prior to storage of the fruit (19; C. L. Xiao, unpublished data). The PCR-based early detection of latent infections by pathogens such as $S$. pyriputrescens and $P$. washingtonensis will enable timely application of fungicides for the control of these diseases.

\section{Literature Cited}

1. Chilvers, M. I., du Toit, L. J., Akamatsu, H., and Peever, T. L. 2007. A realtime, quantitative PCR seed assay for Botrytis spp. that cause neck rot of onion. Plant Dis. 91:599-608.

2. Garibaldi, A., Bertetti, D., Amatulli, M. T., and Gullino, M. L. 2010. First report of postharvest fruit rot in persimmon caused by Phacidiopycnis washingtonensis in Italy. Plant Dis. 94:788.

3. Ghignone, S., and Migheli, Q. 2005. The database of PCR primers for phytopathogenic fungi. Eur. J. Plant Pathol. 113:107-109.

4. Gibson, T., Higgins, D., and Thompson, J. 2009. CLUSTAL W and CLUSTAL X Multiple Sequence Alignment Programs (version 2.0.12, 24th Sept 2009) Available with the program at ftp://ftp.ebi.ac.uk/pub/software/ clustalw2/2.0.12/Readme

5. Ginzinger, D. G. 2002. Gene amplification using real-time quantitative PCR: an emerging technology hits the mainstream. Exp. Hematol. 30:503512.

6. Kim, Y. K., and Xiao, C.L. 2006. A postharvest fruit rot in apple caused by Phacidiopycnis washingtonensis. Plant Dis. 90:1376-1381.

7. Kim, Y. K., and Xiao, C. L. 2007. Infection courts and timing of infection of apple fruit by Sphaeropsis pyriputrescens in the orchard in relation to Sphaeropsis rot in storage. (Abstr.) Phytopathology 97:S57.

8. Kim, Y. K., and Xiao, C.L. 2008. Distribution and incidence of Sphaeropsis rot in apple in Washington State. Plant Dis. 92:940-946.

9. Larkin, M. A., Blackshields, G., Brown, N. P., Chenna, R., McGettigan, P. A., McWilliam, H., Valentin, F., Wallace, I. M., Wilm, A., Lopez, R., Thompson, J. D., Gibson, T. J., and Higgins, D. G. 2007. Clustal W and Clustal X version 2.0. Bioinformatics 23:2947-2948.

10. Liu, Q., and Xiao, C. L. 2009. Infection of 'd'Anjou' pear fruit by Potebniamyces pyri in the orchard in relation to Phacidiopycnis rot during storage. Plant Dis. 93:1059-1064.

11. McCartney, H. A., Foster, S. J., Fraaije, B. A., and Ward, E. 2003. Molecular diagnostics for fungal plant pathogens. Pest Manage. Sci. 59:129-142.

12. Okubara, P.A., Schroeder, K. L., and Paulitz, T.C. 2005. Real-time polymerase chain reaction: applications to studies on soilborne pathogens. Can. J. Plant Pathol. 27:300-313.

13. Pedley, K. F. 2009. PCR-based assays for the detection of Puccinia horiana on chrysanthemums. Plant Dis. 93:1252-1258.

14. Sholberg, P. L., Stokes, S. C., and O'Gorman, D. T. 2009. First report of a new postharvest disease of pear fruit caused by Sphaeropsis pyriputrescens in Canada. Plant Dis. 93:843.

15. Weber, W. S. R. 2011. Phacidiopycnis washingtonensis, Cause of a new storage rot of apples in Northern Europe. J. Phytopathol. 159:682-686.

16. White, T. J., Bruns, T., Lee, S., and Taylor, J. 1990. Amplification and direct sequencing of fungal ribosomal RNA genes for phylogenetics. Pages 315322 in: PCR Protocols. M. A. Innis, D. H. Gelfand, J. J. Sninski, and T. J. White, eds. Academic Press, San Diego, CA

17. Xiao, C. L., and Kim, Y, K. 2008. Postharvest fruit rots in apples caused by Botrytis cinerea, Phacidiopycnis washingtonensis, and Sphaeropsis pyriputrescens. Plant Health Progress. Online publication. doi:10.1094/PHP-20080919-01-DG

18. Xiao, C. L., and Kim, Y. K. 2010. Control of postharvest diseases in apples with reduced-risk fungicides. Stewart Postharvest Rev. Online publication. doi:10.2212/spr.2010.1.6

19. Xiao, C. L., Kim, Y. K., and Boal, R. J. 2011. Control of Sphaeropsis rot in stored apple fruit caused by Sphaeropsis pyriputrescens with postharvest fungicides. Plant Dis. 95:1075-1079.

20. Xiao, C. L., and Rogers, J. D. 2004. A postharvest fruit rot in d'Anjou pears caused by Sphaeropsis pyriputrescens sp. nov. Plant Dis. 88:114-118.

21. Xiao, C. L., Rogers, J. D., Kim, Y. K., and Liu, Q. 2005. Phacidiopycnis washingtonensis - a new species associated with pome fruits from Washington State. Mycologia 97:464-473. 